\title{
Adaptive Space-Frequency Block Coded OFDM
}

\author{
Tae Jin Hwang, Sang Soon Park, and Ho Seon Hwang \\ Department of Electronic Engineering, Chonbuk National University \\ 664-14, Duckjin-Dong 1Ga, Jeonju 561-756, Korea \\ tjhwang@chonbuk.ac.kr
}

\begin{abstract}
This paper discusses an adaptive modulation technique combined with space-frequency block coded OFDM(SFBC OFDM) over frequency selective channels and evaluates the performance in terms of the outdated channel state information(CSI) in mobile environments. This paper employs the Alamouti's diversity scheme in multiple input multiple output OFDM (MIMO OFDM) and an adaptive modulation with enhanced performance. Through the various simulations, the performance of SFBC OFDM employing adaptive modulation is compared with the performance of fixed modulation. Also, in adaptive modulation scheme, the effects of the outdated CSI under mobile environments are shown
\end{abstract}

\section{Introduction}

In order to improve the performance of OFDM system in frequency selective and multipath fading environments, this paper presents an adaptive bit allocation combined with SFBC OFDM. The perfect CSI ensures a desired efficiency/ performance of adaptive modulation scheme. In MIMO OFDM system, by making use of SVD the MIMO channel on each subcarrier is decomposed into parallel noninterfering single input single output(SISO) channels. But, a SFBC OFDM system with Alamouti's diversity scheme in [1] does not require the SVD for the CSI. Assuming the availability of the perfect CSI at the transmitter, the performance gains of adaptive modulation have been demonstrated. This paper examines the impact on performance of an adaptive OFDM system, which combined with SFBC scheme, due to the outdated CSI in mobile fading channel.

\section{Adaptive Space Frequency Block Coded OFDM}

This paper considers a MIMO OFDM system employing the Alamouti's diversity scheme in Fig 1. To begin with, let $\mathbf{H}_{i j}(n)$ be the following diagonal matrix whose diagonal elements are the frequency responses of the channel impulse responses $h_{i j}$ between the $i$-th transmit antenna and the $j$-th receive antenna during the $n$-th time slot

$$
\mathbf{H}_{i j}(n)=\operatorname{diag}\left[H_{i j}(n, 0) \cdots H_{i j}(n, N-1)\right], \quad i=1,2, j=1,2
$$




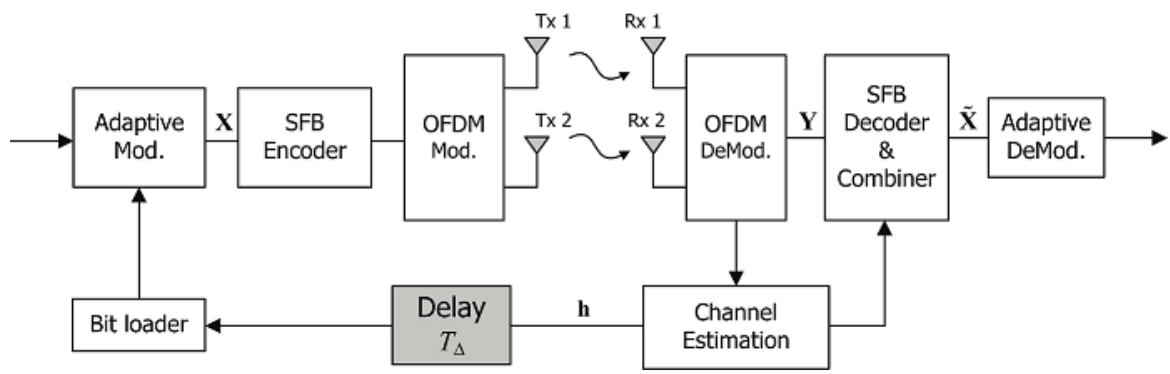

Fig. 1. Block diagram of an adaptive SFBC OFDM

The data symbol vector $\mathbf{X}(n)$ is coded into two vectors $\mathbf{X}_{1}(n)$ and $\mathbf{X}_{2}(n)$ by the space-frequency encoder block as

$$
\begin{aligned}
& \mathbf{X}_{1}(n)=\left[\begin{array}{lllll}
X(n, 0) & -X^{*}(n, 1) & \cdots & X(n, N-2) & -X^{*}(n, N-1)
\end{array}\right]^{T} \\
& \mathbf{X}_{2}(n)=\left[\begin{array}{lllll}
X(n, 1) & X^{*}(n, 0) & \cdots & X(n, N-1) & X^{*}(n, N-2)
\end{array}\right]^{T} .
\end{aligned}
$$

Let $\mathbf{Y}_{j}(n)$ be the $n$-th received OFDM symbol from the $j$-th receive antenna. The receive symbol vector can be represented by the even and odd component vectors as follows

$$
\begin{aligned}
\mathbf{Y}_{j, e}(n) & =\mathbf{H}_{1 j, e}(n) \mathbf{X}_{1, e}(n)+\mathbf{H}_{2 j, e}(n) \mathbf{X}_{2, e}(n)+\mathbf{W}_{j, e}(n), \\
\mathbf{Y}_{j, o}(n) & =\mathbf{H}_{1 j, o}(n) \mathbf{X}_{1, o}(n)+\mathbf{H}_{2 j, o}(n) \mathbf{X}_{2, o}(n)+\mathbf{W}_{j, o}(n), \quad j=1,2 .
\end{aligned}
$$

Assuming the frequency responses between adjacent subcarriers are approximately constant, the combined signals can be rewritten by [2]

$$
\begin{aligned}
& \tilde{\mathbf{X}}_{e}(n)=\left(\left|\mathbf{H}_{11, e}(n)\right|^{2}+\left|\mathbf{H}_{12, e}(n)\right|^{2}+\left|\mathbf{H}_{21, e}(n)\right|^{2}+\left|\mathbf{H}_{22, e}(n)\right|^{2}\right) \mathbf{X}_{e}(n)+\tilde{\mathbf{W}}_{e}(n) \\
& \tilde{\mathbf{X}}_{o}(n)=\left(\left|\mathbf{H}_{11, o}(n)\right|^{2}+\left|\mathbf{H}_{12, o}(n)\right|^{2}+\left|\mathbf{H}_{21, o}(n)\right|^{2}+\left|\mathbf{H}_{22, o}(n)\right|^{2}\right) \mathbf{X}_{o}(n)+\tilde{\mathbf{W}}_{o}(n)
\end{aligned}
$$

In this paper, we consider the bit allocation scheme in [3]. Assume M-QAM is employed for each subcarrier, $b(n, k)$ bits per symbol are sent for the $k$-th subcarrier in the $n$-th OFDM symbol. According to [4][5], given the channel frequency response $H(n, k)$, the instantaneous bit error rate(BER) can be approximated by

$$
P_{e}(n, k)=0.2\left\{-\frac{1.6 \frac{E_{s}}{N_{0}}|H(n, k)|^{2}}{2^{b(n, k)}-1}\right\}
$$

Let us consider the bit allocation in SFBC OFDM system. From the equation (4), the decoupled CSI for bit allocation is as follows

$$
|\mathbf{H}(n, k)|^{2}=\left|H_{11}(n, k)\right|^{2}+\left|H_{12}(n, k)\right|^{2}+\left|H_{21}(n, k)\right|^{2}+\left|H_{22}(n, k)\right|^{2}
$$


By substituting $|\mathbf{H}(n, k)|^{2}$ into $|H(n, k)|^{2}$ in equation (5), the bit allocation for SFBC OFDM is performed and the next procedures for complete bit allocation are based on Chow's method.

\section{Simulation Results}

The parameters of adaptive OFDM system are as follows. Carrier frequency is $2 \mathrm{GHz}$ and the channels bandwidth is $20 \mathrm{MHz}$ which is divided equally among 2048 tones. The channel is based on COST 207 for a hilly terrain area [6] and the SISO channels associated with different couples of transmit/receive antennas are statistically equivalent and independent. The RMS delay spread is $5 \mu \mathrm{s}$. A total of 4096 information bits transmitted in each OFDM frame The velocity of mobile station is $60 \mathrm{~km} / \mathrm{h}$. We allocate $0,2,4$, or 6 bits to each subcarrier. To compare with adaptive OFDM, the conventional OFDM scheme, called as an uniform OFDM, is uniformly modulated by 16-QAM. For the simulation according to feedback delay $T_{\Delta}$, the minimum feedback delay is $81 \mu \mathrm{s}$ and the maximum delay is $810 \mu \mathrm{s}$.

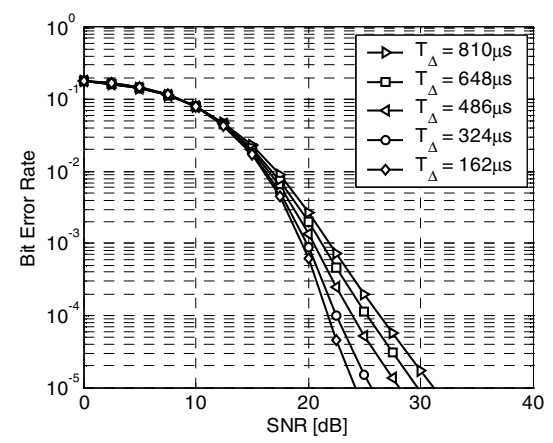

Fig. 2. BER curves of adaptive SFBC OFDM with $2 \mathrm{Tx} / 1 \mathrm{Rx}$ antenna scheme according to feedback delay

Let us consider the question of how the performance of adaptive OFDM system with diversity scheme appears in mobile fading environments. Fig. 2 indicates that the feedback delay has an effect on the performance of adaptive SFBC OFDM with $2 T x / 1 R x$ antenna scheme. As expected, the BER is gradually degraded as the feedback delay increases. This performance degradation is due to unavailability of CSI at the transmission time. Let us examine that an adaptive OFDM system employing spacefrequency block coding scheme is one of ways how to overcome the effects of feedback delay. Fig. 3 indicates the simulation result when the feedback delay is $324 \mu \mathrm{s}$ and $810 \mu \mathrm{s}$, respectively. An adaptive OFDM system with $1 \mathrm{Tx}-1 \mathrm{Rx}$ scheme needs the additional power more than about $7 \mathrm{~dB}$. It is shown that there is no merit of adaptive 1Tx-1Rx OFDM when feedback delay is long. On the other hand, in case of 2Tx-1Rx scheme, adaptive SFBC OFDM systems need the additional power of about $1 \mathrm{~dB}$ and 
$3 \mathrm{~dB}$, respectively. We can see that adaptive OFDM system employing a diversity scheme mitigates the effect of feedback delay. From this result, the performance degradation of adaptive OFDM due to the outdated CSI can be mitigated by diversity technique and we can refer the adaptive 2Tx-2Rx SFBC OFDM as an excellent system which have scarcely power loss in spite of severe feedback delay.

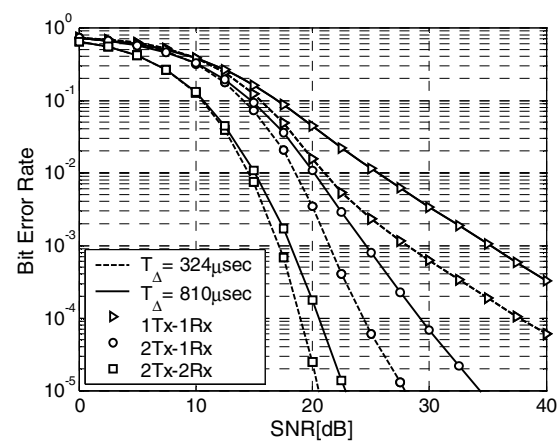

Fig. 3. BER curves according to diversity scheme in case that feedback delays are $324 \mu$ s and $810 \mu$ s respectively

\section{Conclusions}

In this paper combining an adaptive bit allocation scheme with SFBC OFDM system has been discussed. It has been illustrated that the CSI from SVD of MIMO channel is identical to the CSI from SFBC OFDM. From the various simulations, the performance of adaptive SFBC OFDM has been evaluated. Particularly, the BER performances according to the feedback delay have been indicated in detail. In the results, it is very interesting that the diversity schemes mitigate the effect of long feedback delay for adaptive OFDM. Most of all, adaptive SFBC OFDM with 2Tx-2Rx antenna scheme has made an excellent performance in spite of a severe feedback delay.

\section{References}

1. S. M. Alamouti, "A simple transmit diversity technique for wireless communications," IEEE J, Select. Areas Comm., vol. 16, pp. 1451-1458, Oct. 1998.

2. H. Bolcskei and A. Paulraj, "Space-frequency coded broadband OFDM systems," in Proc. Of Wireless Comm. Networking Conf., pp. 1-6. Sept. 2000.

3. P. S. Chow, J. M. Cioffi and J. A. C. Bingham, "A practical discrete multi-ton transceiver allocation algorithm for data transmission over spectrally shaped channels," IEEE Trans. on Comm, vol. 43, pp. 773-775, Apr 1995.

4. S. T. Chung and A. J. Goldsmith, "Degrees of freedom in adaptive modulation: a unified view," IEEE trans. On Comm., vol. 49, pp. 1561-1571, sep. 2001

5. S. Ye, R. S. Blum and L. L. Cimini, "Adaptive modulation for variable-rate OFDM systems with imperfect channel information," Proc, VTC 2002, pp. 767-771.

6. M. Patzold, Mobile Fading Channels, Wiley, 2002. 